\title{
Distance scale, variable stars and stellar populations in Local Group galaxies
}

\author{
G. Clementini ${ }^{1}$, L. Baldacci ${ }^{2}$, A. Bragaglia ${ }^{1}$, E. Carretta ${ }^{3}$, \\ L. Di Fabrizio ${ }^{4}$, R.G. Gratton ${ }^{3}$, C. Greco ${ }^{2}$, M. Gullieuszik ${ }^{5}$, E.V. Held ${ }^{3}$, \\ M. Maio ${ }^{1}$, M. Marconi ${ }^{6}$, F. Matonti ${ }^{2}$, Y. Momany ${ }^{5}$, E. Poretti ${ }^{7}$, \\ L. Rizzi ${ }^{5}$, I. Saviane ${ }^{8}$, E. Taribello ${ }^{2}$ \\ ${ }^{1}$ INAF - Bologna Observatory, via Ranzani 1, I-40127 Bologna \\ ${ }^{2}$ Department of Astronomy, University of Bologna, via Ranzani 1, \\ I-40127 Bologna \\ ${ }^{3}$ INAF - Padua Observatory, vicolo dell'Osservatorio 5, I-35122 Padova \\ ${ }^{4}$ INAF - Centro Galileo Galilei \& Telescopio Nazionale Galileo, PO Box \\ 565, 38700 Santa Cruz de La Palma, Spain \\ ${ }^{5}$ Department of Astronomy, University of Padua, vicolo \\ dell'Osservatorio 2, I-35122 Padova \\ ${ }^{6}$ INAF - Capodimonte Observatory, via Moiariello 16, I-80131 Napoli \\ ${ }^{7}$ INAF - Brera Observatory, via Bianchi 46, I-23807 Merate \\ ${ }^{8}$ European Southern Observatory, Casilla 19001, Santiago 19, Chile
}

\begin{abstract}
We present an overview of our study of the short period variable stars in the Large Magellanic Cloud, and in the dwarf galaxies Fornax, Leo I, and NGC 6822. Light curves are presented for RR Lyrae stars, Anomalous Cepheids and, for the first time, for Dwarf Cepheids in the field and in the globular cluster \#3 of the Fornax galaxy.
\end{abstract}

\section{Introduction}

Pulsating variable stars play a fundamental role in establishing the astronomical distance scale, tracing different stellar populations, and studying radial trends, star formation history and formation mechanisms of the host galaxy. In recent years we have assembled a database on the short period variable stars $(P<4 \mathrm{~d})$ in a number of Local Group galaxies. Important assets of our study were: (1) the collection of both photometric and spectroscopic data with wide field imagers and large aperture telescopes (e.g. the Wide Field Imager - WFI of the ESO/MPI 2.2-m and the ESO Very Large Telescopes - VLTs) (2) the use of DAOPHOT and ALLFRAME (Stetson 1994) reduction packages, and (3) the detection of the variable stars with the Image Subtraction method (ISIS2.1, Alard 2000). Here we briefly present results for 4 of the galaxies in our sample, 

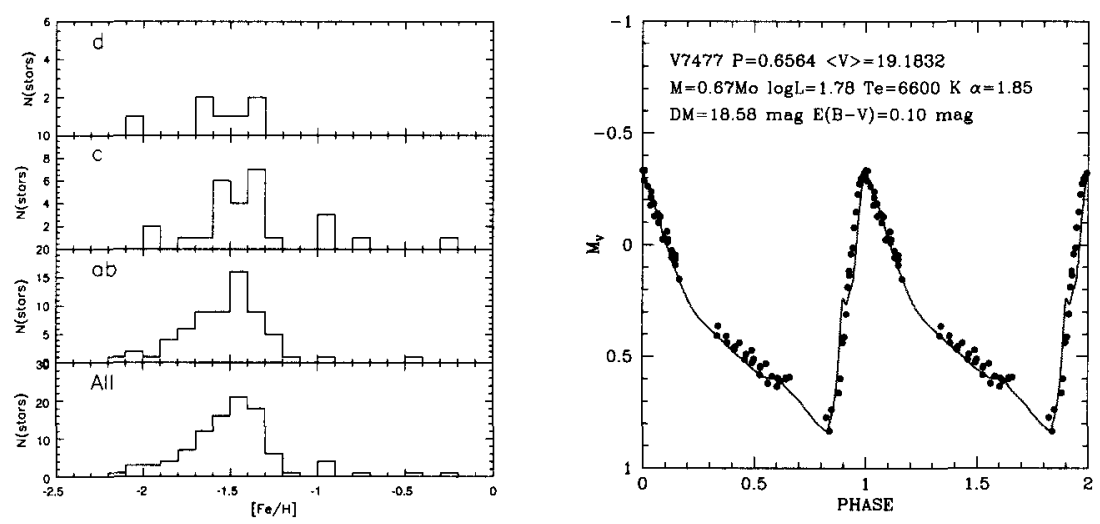

Figure 1. Left panel: Metallicity distribution of double-mode $(d-)$, first overtone $(c-)$, and fundamental mode ( $a b$-type) RR Lyrae stars in the LMC; the bottom panel shows the total sample (from Gratton et al. 2003, in preparation). Right panel: Theoretical modelling of the $V$ light curve of the LMC field RR Lyrae star V7477 (from Marconi \& Clementini 2003, in preparation).

namely: the Large Magellanic Cloud (LMC), Leo I, NGC 6822 and Fornax. A global description of the project can be found in Clementini (2003).

\section{The LMC: the zeropoint of the astronomical distance scale}

Being the galaxy much closer to the Milky Way, the LMC is the first step of the extragalactic distance scale. Due to its complex stellar population several distance indicators of both Population I and II are present in this galaxy, thus allowing a direct comparison of the distance scales they provide.

Our LMC variable star sample includes 125 RR Lyrae stars (RRLs), 4 Anomalous Cepheids (ACs), 11 Cepheids, 11 binaries, and $1 \delta$ Scuti star located close to the LMC bar. We have obtained $B V I$ photometry accurate to 0.02 mag (at the luminosity level of the LMC RRLs) for the full sample, and individual spectroscopic metallicities (with FORS1 at the VLT) for about $80 \%$ of the variables (Gratton et al. 2003, in preparation). The metallicity distribution of the RRLs is shown in the left panel of Fig. 1. Our data allowed (i) a very accurate definition of the average apparent magnitude of the LMC RRLs: $V_{0}$ (RR) $=19.064 \pm 0.064$, (ii) the estimate of the local reddening from the pulsational properties of these variables: $\langle E(B-V)\rangle=0.10 \pm 0.02$, and (iii) the definition of the slope of the RRLs luminosity-metallicity relation: $\Delta M_{V}(\mathrm{RR}) / \Delta[\mathrm{Fe} / \mathrm{H}]=0.214 \pm 0.047$. The corresponding distance modulus of the LMC from the average of several independent Population I and II distance indicators is: $\mu_{\mathrm{LMC}}=18.515 \pm 0.085$, (Clementini et al. 2003a). An independent estimate of the LMC distance was also obtained by fitting the observed RRL light curves with the theoretical pulsational models by Bono et al. (2003). An 
example of this theoretical modelling is shown in the right panel of Fig. 1. The distance modulus derived for this star is $18.58 \pm 0.10$ (Marconi \& Clementini 2003 , in preparation).

\section{Leo I and NGC 6822: radial trends and star formation history}

RRLs, ACs and Classical Cepheids allow the unambiguous tracing of the old $(t \gtrsim 10 \mathrm{Gyr})$, intermediate age $(t \simeq 1-5 \mathrm{Gyr})$ and young $(t \lesssim 100 \mathrm{Myr})$ stellar populations across the central regions of the parent galaxies. We have mapped the Leo I dwarf spheroidal galaxy (dSph) with the 8 CCDs of the WFI mosaic. We discovered a conspicuous population of RRLs extending all the way to the centre of the galaxy (Held et al. 2001), and about 40 candidate ACs more concentrated in the inner regions of Leo I (see Figure 1 of Baldacci et al. 2003a; Clementini et al. 2004, in preparation). Our finding confirms that a first burst of stellar formation involving the whole galaxy occurred in Leo I about 10-13 Gyr ago, while the subsequent star formation episodes that generate the candidate ACs were more confined toward the galaxy centre.

Time series photometry of the dwarf irregular galaxy NGC 6822 was obtained with FORS2 at the VLT. We discovered about 450 candidate variables among which 17 RRLs and 20 brighter short-period, small amplitude variables, that we named Low Luminosity (LL) Cepheids (Clementini et al. 2003b; Baldacci et al., these proceedings). The classical instability strip in the colourmagnitude diagram of NGC 6822 appears to be uniformly filled from the RRLs up to the Classical Cepheids (Baldacci et al. 2003b; Held et al. 2004, in preparation), thus reflecting the extended star formation occurring in this galaxy.

\section{Fornax: galaxy formation mechanisms}

In merging scenarios the Galactic halo was assembled by accreted protogalactic fragments resembling the present-day dwarf spheroidal satellites of the Milky Way (MW). A number of the Galactic globular clusters (GGCs) may originate from these disrupted dSphs and should keep imprinted the characteristics of their "ancestors". A marked feature of the GGCs is their division into two sharply distinct Oosterhoff groups (Oosterhoff 1939), however no division in Oosterhoff types is observed in the present-day dSphs (see Catelan, these proceedings). Besides Sagittarius, Fornax is the only LG dSph known to host globular clusters. We observed a WFI area of the galaxy that hosts the globular cluster Fornax 3. A sample of 108 RRLs (among which there are 20 double-mode candidates), 19 ACs and 25 Dwarf Cepheids (DCs) were identified in the chip of the WFI mosaic that contains cluster \#3. Respectively 27, 8 and 2 of these variables belong to the cluster. The left panel of Fig. 2 shows the colour-magnitude diagram of Fornax from data in this chip. The classical instability strip is filled from the DCs up to the ACs. The right panel shows light curves of variables in Fornax 3 (Greco 2003). The average period of the $a b$-type RRLs is $0.595 \mathrm{~d}$ for the field variables, and $0.612 \mathrm{~d}$ in Fornax 3 . This $\left\langle P_{a b}\right\rangle$ value places Fornax 3 at the lower edge of the distribution of the Oosterhoff type II GGCs. 

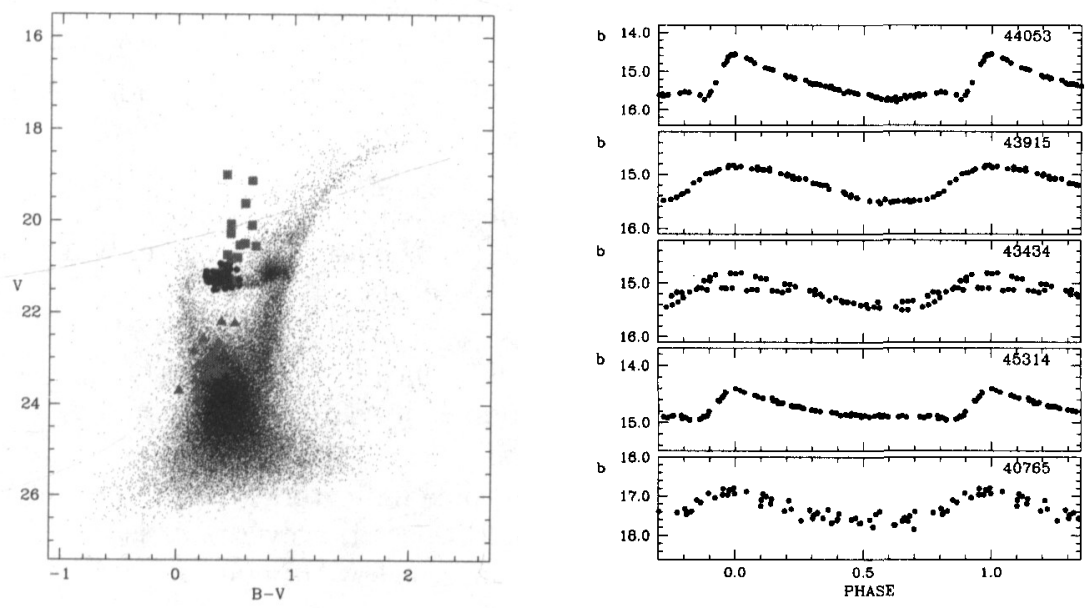

Figure 2. Left panel: Colour-magnitude diagram of Fornax (from data in chip 6) with DCs, RRLs and ACs marked by triangles, circles and squares, respectively. Right panel: $b$ instrumental light curves of $a b-, c-$ and $d$-type RRLs, an AC, and a DC in Fornax 3 (from Greco 2003).

\section{References}

Alard, C. 2000, A\&AS, 144, 363

Baldacci, L., Matonti, F., Clementini, G., Rizzi, L., Held, E.V., Di Fabrizio, L., Momany, Y., Saviane, I. 2003a, in Stars in Galaxies, ed. M. Bellazzini, A. Buzzoni \& S. Cassisi, Mem. S. A. It, in press (astro-ph/0305506)

Baldacci, L., Clementini, G., Held, E.V., Rizzi, L. 2003b, in Variability with wide-field imagers, ed. G. Bono, M. Castellani \& D. Trevese, Mem. S. A. It., Vol. 74, p. 860 (astro-ph/0303094)

Bono, G., Caputo, F., Castellani, V., Marconi, M., Storm, J., Degl'Innocenti, S. 2003, MNRAS, 344, 1097

Clementini, G. 2003, in Variability with wide-field imagers, ed. G. Bono, M. Castellani \& D. Trevese, Mem. S. A. It., Vol. 74, p. 878 (astro$\mathrm{ph} / 0303067$ )

Clementini, G., Gratton, R.G., Bragaglia, A., Carretta, E., Di Fabrizio, L., Maio, M. 2003a, AJ, 125, 1309

Clementini, G., Held, E.V., Baldacci, L., Rizzi, L. 2003b, ApJ, 588, L85

Greco, C. 2003, Master of Science Thesis, Univ. of Bologna

Held, E.V., Clementini, G., Rizzi, L., Momany, Y., Saviane, I., Di Fabrizio, L. 2001, ApJ, 562, L39

Oosterhoff, P.Th. 1939, The Observatory, 62, 104

Stetson, P.B. 1994, PASP, 196, 250 


\section{Discussion}

Guinan: Is the quality of the photometry of eclipsing binaries good enough to derive their orbital and physical properties?

Clementini: Yes, I believe so. The photometric accuracy of the light curves for the eclipsing binaries in our sample is about $0.02 \mathrm{mag}$. However, only a couple of the binaries in our sample are detached systems.

Jurcsik: There seems to be a systematic difference between the LMC distances from Population I and II distance indicators. Is it real?

Clementini: Indeed, some of the Pop. I distance indicators give distance moduli for the LMC slightly longer than the Pop. II ones. However, this is not true of the clump method, and in any cases differences are within one sigma.

Daszynska: How did you determine the metallicities of these stars? Which models did you use?

Clementini: Metallicities were determined using a revised version of the $\Delta \mathrm{S}$ method (Preston 1959, ApJ, 130, 507). In this technique abundances of RR Lyrae stars are obtained by comparing the strength of the Ca II K line with that of the $\mathrm{H}$ lines. We derived our metallicities by comparing the instrumental fluxes in a few spectral bands centred on the CaII K line, $\mathrm{H} \delta, \mathrm{H} \gamma$ and $\mathrm{H} \beta$, obtained by simply integrating the spectra of the LMC RR Lyrae stars within given limits, to those of RR Lyrae and horizontal branch stars in a number of calibrating Galactic globular cluster of known metal abundance, that had been observed with the same instrumental set-up of the program stars. No model atmospheres were directly involved in our analysis.

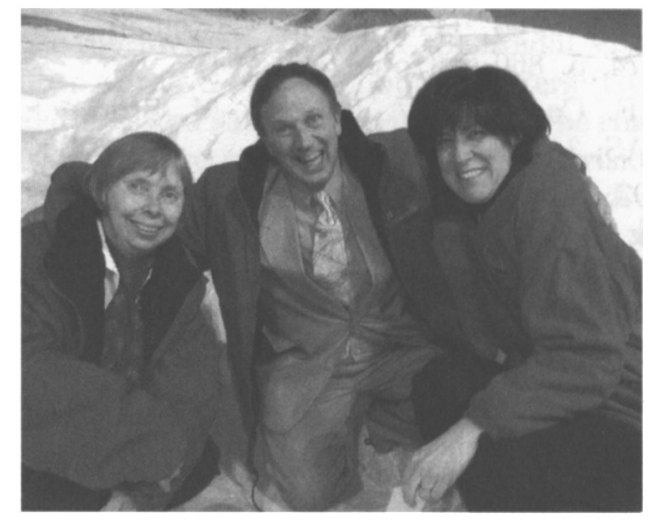

Nancy Evans, Ed Guinan and Gisella Clementini 